\title{
Targeting self-renewal of cancer stem cells in colorectal cancer-a future treatment option?
}

A recent study published in Nature Medicine has shown that targeting the self-renewal property of cancer stem cells could have potential as a therapeutic approach in colorectal cancer.

"We first identified that colon cancer has cancer stem cells (CSCs) in 2007, and since then we have been interested in understanding what distinguishes CSCs from non-CSCs," explains John Dick, corresponding author of the study. The capacity for self-renewal is one of the key unique features of CSCs. BMI-1 is one of the best known regulators of self-renewal in stem cells, but its activity had not been looked at in colorectal cancer specifically.

First, the researchers used genetic approaches to investigate the role of BMI-1 in colorectal cancer. Using in vitro and in vivo methods, they were able to show that downregulation of BMI1 reduced human colorectal cancer growth and impaired tumour initiation.

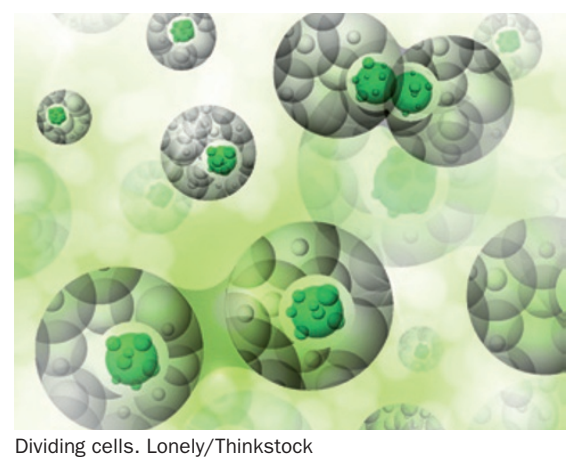

tumour growth in a preclinical model of primary human colorectal cancer.

Given that colorectal cancer is the third leading cause of cancer-related death in the West, and that tumour recurrence is a major clinical challenge, the findings of this study are potentially very exciting. The results show that targeting a hallmark biological property of stem cells, that is, their capacity for self-renewal, could be clinically relevant.

"From now on, it is important to develop

The researchers then went on to investigate the effects of targeting BMI-1 by small-molecule inhibition. "The good fortune was that Catherine O'Brien met Tom Davis at a cancer meeting and realized that they had a small molecule targeting BMI-1, something no-one else had done," explains Dick. The drug worked as expected-BMI-1 inhibition resulted in irreversible reduction in the numbers of colorectal CSCs and impairment of even better and more effective drugs that can be used in patients, and then test them for toxicity in phase I trials," concludes Dick. The researches also suggest that future studies could target other key pathways driving self-renewal in CSCs.

Isobel Leake

Original article Kreso, A. et al. Self-renewal as a therapeutic target in human colorectal cancer. Nat. Med. doi: $10.1038 / \mathrm{nm} .3418$ 